\title{
Management of Pregnancy in Rheumatic Disease
}

The Editor's Pick is the review paper by Maguire et al. Managing patients with rheumatic musculoskeletal disorders (RMD) during

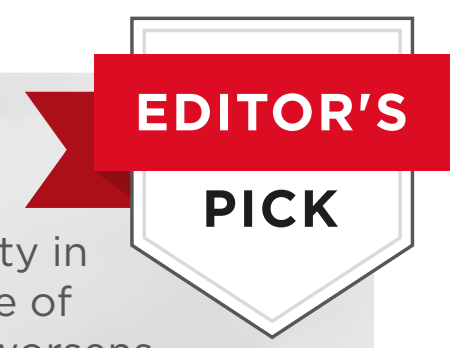
pregnancy and the postpartum period is challenging. Disease activity in some RMDs, such as rheumatoid arthritis, improve during the course of pregnancy and in others, such as systemic lupus erythematosus, it worsens. Then there is the issue of drug treatment during pregnancy to maintain remission and the potential undesirable effects on the fetus. This complex subject area is reviewed as well as the current American College of Rheumatology (ACR) guidelines on pregnancy and RMDs.
Authors:
*Sinead Maguire, ${ }^{1,2}$ Finbar O’Shea ${ }^{1,2}$
1. Department of Rheumatology, St James' Hospital, Dublin, Ireland
2. School of Medicine, Trinity College Dublin, Ireland
*Correspondence to sinead.magu@gmail.com

Disclosure:

The authors have declared no conflicts of interest.

Received:

06.02 .21

Accepted:

16.04.21

Keywords:

Connective tissue disease, females' health, inflammatory arthritis, pregnancy, pregnancy planning, reproductive health, rheumatic disease (RMD), vasculitis.

Citation:

EMJ Rheumatol. 2021;8[1]:86-93.

\section{Abstract}

Managing patients with rheumatic diseases (RMD) during pregnancy and the postpartum period can be a challenge for both rheumatologists and obstetricians. While disease activity during the course of pregnancy varies with regard to the presence of underlying conditions, maintenance of remission from conception through to delivery increases the chances of an uncomplicated pregnancy. A period of remission of at least 6 months prior to conception increases the chance of a successful conception while decreasing the risk of flares during pregnancy. For this reason, discussion of pregnancy in females with RMDs should begin prior to conception with risk stratification and pregnancy planning. This allows for the transfer to pregnancy-compatible medications, disease stabilisation, determination of autoantibody status, and evaluation of end-organ damage. During pregnancy, where possible, disease activity should be monitored with scores modified to allow use in pregnancy. Prompt recognition and treatment of active disease is essential to minimise the risk to the pregnancy. Systemic lupus erythematosus and axial spondyloarthropathy can present diagnostic dilemmas due to overlap of symptoms of disease activity and normal pregnancy. Patients with end-organ involvement, such as systemic lupus erythematosus or systemic sclerosis, face a higher risk of adverse pregnancy outcomes and disease progression. Close monitoring of patients with RMD should be done by both obstetrics and rheumatology, with regular communication between specialties. Medications should be reviewed at each stage of pregnancy to ensure compliance with the current American College of Rheumatology (ACR) guidelines and the adequate treatment of RMDs. 


\section{INTRODUCTION}

Many patients with rheumatic diseases (RMD) are diagnosed during their childbearing years. ${ }^{1}$ Historically, females with RMDs were advised to avoid pregnancy due to potential risk to both mother and fetus. ${ }^{2}$ Because of advances in detection of disease activity and therapeutics, this is no longer the case and most females with RMDs can safely become pregnant; ${ }^{3}$ however, they may face an increased risk of a number pregnancy-related complications and require closer monitoring during their pregnancy. ${ }^{4}$

Pregnancy with RMD should involve a period of planning between the patient and rheumatologist prior to actively trying to conceive. ${ }^{5,6}$ The primary objective in managing a pregnancy is both a healthy mother and child. In order to ensure favourable outcomes, a number of factors, including medication usage, disease activity, and antibody status, must be evaluated and managed (Table 1). ${ }^{7}$ Pregnancy planning to risk assess and address these issues allows the rheumatologist to help their patients safely navigate both the pregnancy and postpartum period.

During pregnancy, the female body undergoes a series of significant physiological and hormonal changes, which can affect disease activity. $^{8}$ During pregnancy, these patients can pose a challenge to rheumatologists as flares can be confused with complications or physiological changes of pregnancy. Advances in understanding of the disease processes has helped differentiate between these processes. Level of inflammation at the time of conception has been shown to directly impact pregnancy outcome, making control of disease activity essential.9,10 A number of disease activity scores have been modified for use in pregnancy; however, most are focused around rheumatoid arthritis (RA) and systemic lupus erythematosus (SLE), with a considerable paucity of tools for other RMDs." For this reason, close monitoring by both rheumatology and obstetrics is necessary during the course of pregnancy.

\section{SEARCH STRATEGY}

For this review, PubMed, Cochrane, and EMBASE were searched for articles related to pregnancy in RMD, with more specific searches for each RMD. ${ }^{12}$ Preference was given to more recently published literature to reflect most recent research. Bibliographies of included articles were also searched for additional sources.

Table 1: Factors of rheumatic diseases involved in pregnancy outcome.

\begin{tabular}{|l|l|}
\hline Positive impact & Negative impact \\
\hline Pregnancy planning and counselling & $\begin{array}{l}\text { Active disease§ } \\
\text { Eisease remission* }\end{array}$ \\
Known autoantibody status & $\begin{array}{l}\cdot \quad \text { Lupus nephritis } \\
\text { Medication adherence }\end{array}$ \\
Use of pregnancy-safe medications ${ }^{\dagger}$ & Unplanned pregnancy \\
Close monitoring of disease activity $\ddagger$ & Unplanned cessation of treatment \\
Optimisation of general health & \\
\hline
\end{tabular}

*Remission of disease for at least 6 months prior to conception and throughout pregnancy.

+Adjusted for each stage of pregnancy.

¥With outcome measures adjusted for pregnancy where possible.

§Both prior to conception and during pregnancy.

**Without consultation with a rheumatologist due to risk of disease activity. 


\section{PRIOR TO CONCEPTION}

\section{Counselling and Planning}

For females with RMDs, pregnancy planning is an essential component of pregnancy in the months prior to conception. The importance of this period of planning and counselling in RMD cannot be understated. Lack of pregnancy planning in the general population has been associated with increased incidence of low birth weight and even neonatal mortality. ${ }^{13,14}$ Unplanned pregnancies are also associated with increased rates of smoking, stress, and lack of prenatal care. ${ }^{15}$ This is also seen in patients with RMDs but conception is further complicated by risk of active disease and use of medications not compatible with pregnancy.

Recent studies in SLE demonstrated that lack of planning for pregnancy was a significant predictor of adverse pregnancy outcomes. ${ }^{16}$

This issue is correctable with improved patient education and increased physicianled discussion of family planning during rheumatology consultations. ${ }^{17}$ Barriers to these conversations discussed in a SLE focus group include busy clinic schedules and difficulty initiating pregnancy conversations. ${ }^{18}$ However, this group also highlighted the disconnect in patient and physician risk perception of pregnancy, which could be remedied by open conversations about pregnancy as part of regular rheumatology consultations.

\section{Disease Control}

Control of maternal disease during pregnancy is associated with improved pregnancy outcomes and lower risk of fetal complications. ${ }^{5,9}$ The importance of disease control begins in the pre-conception stage, as females with active disease are advised to wait for remission prior to actively trying to conceive. ${ }^{6,7}$ Patients planning for pregnancy should be in remission for at least 6 months prior to conception. ${ }^{10}$ Patients with significant end-organ damage should be counselled on their risk stratification prior to conception. ${ }^{19}$ In cases of severe disease, including advanced pulmonary arterial hypertension (PAH), chronic renal impairment Stage IV/V, or in cardiac failure, there is considerable risk of maternal morbidity and mortality. In such cases, individuals should be advised against pregnancy (Table 2). ${ }^{10,17,20}$

Table 2: Relative and absolute contraindications to pregnancy in patients with rheumatic diseases. ${ }^{12,19,21}$

\begin{tabular}{|l|l|}
\hline Relative contraindications & Absolute contraindications \\
\hline Severe SLE flare within the past 6 months* & Advanced pulmonary hypertension \\
Pulmonary hypertension & End-stage renal disease \\
Severe restrictive lung disease & Severe cardiac failure \\
Moderate to severe cardiac failure & \\
Severe valvular disease & \\
CKD stage 4-5 & \\
Uncontrolled hypertension & \\
Previous early-onset pre-eclampsia or HELLP+ & \\
\hline
\end{tabular}

*Including renal flare.

+Despite therapy with aspirin and heparin

CKD: chronic kidney disease; HELLP: haemolysis, elevated liver enzymes, and low platelets. 
Table 3: Summary of the American College of Rheumatology (ACR) guidelines on medication for rheumatic disease usage during conception, pregnancy, and lactation. ${ }^{9}$

\begin{tabular}{|c|c|c|c|}
\hline \multicolumn{4}{|l|}{ Conception } \\
\hline Safe to continue & $\begin{array}{l}\text { Conditionally recommend } \\
\text { to continue }\end{array}$ & Stop prior to conception & Unknown \\
\hline $\begin{array}{l}\text { Hydroxychloroquine } \\
\text { Sulfasalazine } \\
\text { Colchicine } \\
\text { Azathioprine } \\
\text { Certolizumab pegol } \\
\text { Cyclosporine } \\
\text { Tacrolimus }\end{array}$ & $\begin{array}{l}\text { Prednisolone } \\
\text { Cyclosporine } \\
\text { NSAIDs } \\
\text { Infliximab } \\
\text { Etanercept } \\
\text { Adalimumab } \\
\text { Golimumab } \\
\text { Rituximab } \\
\text { Anakinra } \\
\text { Belimumab }^{b} \\
\text { Abatacept }^{b} \\
\text { Tocilizumab }^{b} \\
\text { Secukinumab }^{b} \\
\text { Ustekinumab }^{b}\end{array}$ & $\begin{array}{l}\text { Methotrexate } \\
\text { Leflunomide } \\
\text { Mycophenolate mofetil } \\
\text { Cyclophosphamide } \\
\text { Thalidomide }\end{array}$ & $\begin{array}{l}\text { Tofacitinib } \\
\text { Apremilast } \\
\text { Baricitinib }\end{array}$ \\
\hline \multicolumn{4}{|l|}{ Pregnancy } \\
\hline Safe to continue & $\begin{array}{l}\text { Conditionally recommend } \\
\text { to continue }\end{array}$ & Stop prior to conception & Unknown \\
\hline $\begin{array}{l}\text { Hydroxychloroquine } \\
\text { Sulfasalazine } \\
\text { Colchicine } \\
\text { Azathioprine } \\
\text { Certolizumab pegol } \\
\text { Cyclosporine } \\
\text { Tacrolimus }\end{array}$ & $\begin{array}{l}\text { Prednisolone }^{a} \\
\text { Cyclosporine } \\
\text { NSAIDs }^{c} \\
\text { Infliximab }^{d} \\
\text { Etanercept }^{d} \\
\text { Adalimumab }^{d} \\
\text { Golimumab }^{d} \\
\text { Cyclophosphamide }^{e} \\
\text { Rituximab }^{f}\end{array}$ & $\begin{array}{l}\text { Methotrexate } \\
\text { Leflunomide } \\
\text { Mycophenolate mofetil } \\
\text { Thalidomide } \\
\text { Anakinrag } \\
\text { Belimumab9 }^{g} \\
\text { Abatacept } \\
\text { Tocilizumabg }^{9} \\
\text { Secukinumabg } \\
\text { Ustekinumabg }\end{array}$ & $\begin{array}{l}\text { Tofacitinib } \\
\text { Apremilast } \\
\text { Baricitinib }\end{array}$ \\
\hline \multicolumn{4}{|l|}{ Lactation } \\
\hline $\begin{array}{l}\text { Hydroxychloroquine } \\
\text { Sulfasalazine } \\
\text { Colchicine } \\
\text { Certolizumab pegol } \\
\text { Infliximab } \\
\text { Etanercept } \\
\text { Adalimumab } \\
\text { Golimumab } \\
\text { Rituximab }\end{array}$ & $\begin{array}{l}\text { Azathioprine } \\
\text { Prednisolone }^{\text {h }} \\
\text { Cyclosporine }^{\text {h }} \\
\text { Tacrolimush }^{\text {h }} \\
\text { NSAIDs }^{j} \\
\text { Anakinrak }^{k} \\
\text { Belimumabk }^{\text {Abataceptk }} \\
\text { Tocilizumabk }^{k} \\
\text { Secukinumabk }^{\text {Ustekinumabk }}\end{array}$ & $\begin{array}{l}\text { Methotrexatel' } \\
\text { Leflunomide } \\
\text { Mycophenolate mofetil } \\
\text { Cyclophosphamide } \\
\text { Thalidomide }\end{array}$ & $\begin{array}{l}\text { Tofacitinibm }^{m} \\
\text { Apremilast }^{m} \\
\text { Baricitinib }^{m}\end{array}$ \\
\hline
\end{tabular}

a Minimise dose to $<20 \mathrm{mg} /$ day with use of alternative pregnancy-compatible immunosuppressant.

${ }^{b}$ Discontinue at conception.

'Avoid after 32 weeks to prevent premature closure of PDA.

${ }^{d}$ Discontinue in the third trimester several half-lives prior to delivery.

e Only to be used in life- or organ-threatening disease, in the second or third trimester only.

${ }^{f}$ Life- or organ-threatening disease only. 
${ }^{f}$ Life- or organ-threatening disease only.

${ }^{g}$ Conditionally recommend to discontinue in pregnancy.

hLow transfer in breastmilk.

'In doses $>20$ mg, breastfeeding should be delayed by 4 hours following consumption.

ipreferably use ibuprofen when needed.

${ }^{k}$ No data currently available but due to large size of molecule, minimal transfer in breastmilk anticipated.

'Limited data available, which suggests low transfer in breastmilk.

mNo data available but small size of molecule suggests transfer into breastmilk likely.

NSAIDs: Non-steroidal anti-inflammatory drugs; PDA: patent ductus arteriosus.

\section{Medication}

One of the main causes of patient and physician concern during pregnancy is around medication usage. Certain medications used to treat RMDs are not safe in pregnancy; 22 however, this is not true for all medications as outlined by the American College of Rheumatology (ACR) guidelines (Table 3). ${ }^{7}$ Despite this, many females with RMDs stop their RMD medication prior to conception or in the early stages of pregnancy. ${ }^{23}$ Unfortunately, this increases their risk of flare, which requires rescue treatment with steroids followed by a period of unstable disease while their disease-modifying anti-RMD medication is restarted. ${ }^{21}$ Open discussion with patients with RMD planning to conceive around pregnancysafe medication, including the risks and benefits of stopping medication, could aid in limiting such situations.

This could also be an opportunity to ensure that the patient has commenced a prenatal vitamin. Females on folic acid antagonists including sulfasalazine will require higher doses of folic acid of $5 \mathrm{mg} /$ day when trying to conceive through to the completion of the first trimester to decrease risk of neural tube defects. ${ }^{24}$

Many people with RMDs are treated with medications that are not compatible with pregnancy or have limited data for use in pregnancy. ${ }^{22}$ For these patients, a period of transition to pregnancy-safe medications should take place prior to conception. It is important to remind those taking medications such as methotrexate and mycophenolate mofetil that these medications are not safe in pregnancy and to remind of the importance of using reliable contraception.

\section{Autoantibody Status}

Assessment for the presence of autoantibodies such as anti-Ro/SS-A, anti-La/SS-B, and antiphospholipid status can determine pregnancy risk and need for additional medication during conception and pregnancy. Presence of anti-Ro or anti-La antibodies is associated with risk of fetal congenital heart block and development of neonatal lupus. Treatment of antibody-positive females during pregnancy with hydroxychloroquine significantly reduces this risk. ${ }^{25}$

Patients with SLE, history of recurrent miscarriage, or other severe pregnancy outcomes should be evaluated for presence of antiphospholipid antibodies (aPL). ${ }^{20}$ This includes screening for $\beta-2$ glycoprotein 1, lupus anticoagulant, and anti-cardiolipin antibodies. Use of low-dose aspirin is recommended for patients with $\mathrm{aPL}$ to decrease risk of preeclampsia in pregnancy. ${ }^{26}$ Once pregnant, patients with a previous history of obstetric or thrombotic antiphospholipid syndrome (APS) will require therapeutic or prophylactic lowmolecular-weight or unfractionated heparin depending on their risk stratification.,27

\section{PREGNANCY}

During the course of pregnancy, the female body undergoes a series of physiologic changes driven by dramatic alteration of hormone levels. These changes have varying effect on disease activity in RMD. Monitoring for signs of active disease is an essential component of care for the patient who is pregnant with RMD; however, accuracy of conventional tools to detect active disease can be limited by physiologic changes of pregnancy. An article outlining these challenges highlighted that a number of instruments have 
been developed to more accurately assess disease activity in RMD pregnancies."

While there are no specific guidelines for how often these patients should be seen by a rheumatologist during pregnancy, clinical assessment once per trimester allows regular monitoring of disease activity. More frequent monitoring may be required in cases of active disease, pregnancy complications, or underlying end-organ damage. Joint care of patients with RMD who are pregnant and regular communication between obstetrics and rheumatology can help to safely manage these challenging cases.

\section{Systemic Lupus Erythematosus}

Pregnancy in SLE is commonly encountered as this disease has a strong female predominance, with many diagnosed during their childbearing years. SLE can be difficult to monitor and manage due to the multi-organ involvement of autoimmune disease. This is further complicated by physiological changes of pregnancy, which can mimic disease activity, such as observed increases in erythrocyte sedimentation rate ${ }^{28}$ or development of thrombocytopenia. ${ }^{29}$ Other common pregnancy issues, including fatigue and proteinuria, can also be signs of active disease and confuse measures of disease monitoring. However, pregnancy has not been shown to affect presence of autoantibodies.

Disease activity is the most important predictor of pregnancy outcome in SLE, with $23.5 \%$ of patients experiencing active disease during pregnancy. ${ }^{30}$ Pattern of organ involvement prior to conception accurately predicts organ involvement in pregnancy flares and can be helpful for targeted monitoring of disease activity. ${ }^{31}$ There are a number of SLE scores that have been adapted for pregnancy including SLE Pregnancy Disease Activity Index (SLEPDAI), ${ }^{32}$ Systemic Lupus Activity Measure (SLAM)-Revised, Lupus Activity Index in Pregnancy (LAI-P), ${ }^{33}$ and the British Isles Lupus Assessment Group (BILAG) 2004 for pregnancy. ${ }^{34}$

Patients with lupus nephritis or renal impairment have an increased risk of further renal deterioration and adverse pregnancy outcomes. ${ }^{35}$ This risk remains even in patients with previous lupus nephritis in remission at the time of conception. ${ }^{4}$ Patients should be assessed for presence of hypertension, proteinuria, and raised serum creatinine $>100 \mu \mathrm{mol} / \mathrm{L}$ routinely because of association with increased risk of adverse outcomes. ${ }^{36}$ Risk for renal flare during pregnancy is associated with active renal disease at the time of conception, with a relative risk of 9.0 in cases of non-remission and a relative risk of 3.0 in partial remission. ${ }^{37}$ To reduce risk of flare, disease activity should be controlled throughout pregnancy. This involves use of immunosuppressive medication, adequate blood pressure control, and use of lowdose aspirin to decrease risk of pre-eclampsia. ${ }^{38}$ Renin-angiotensin blockers should be stopped prior to pregnancy or during the first trimester and changed to alternative hypertensive therapy.

Onset of SLE can occur during the course of pregnancy in patients with no previous history of connective tissue disease. Such cases have higher rates of thrombocytopenia, pregnancy loss, and active disease in pregnancy as compared to those with a previous diagnosis. ${ }^{39}$ Higher rates of adverse maternal complications have also been seen in this population. ${ }^{40}$ Prompt recognition and treatment is critical but can be a challenge due to the overlap of symptoms with that of normal pregnancy. Monitoring levels of complement and anti-doublestranded DNA antibodies can identify new-onset SLE in a patient who is pregnant. Certain clinical features, such as onset of renal impairment and hypertension prior to 20 weeks' gestation, are suggestive of SLE rather than pre-eclampsia. ${ }^{17}$

Autoantibody status is crucial for management of SLE pregnancies. In patients with SLE who have not had antibody status assessed prior to conception, this should be done as soon as possible in pregnancy along with an ECG. In cases of corrected QT prolongation or previous history of cardiac involvement, a baseline echocardiogram is useful to assess cardiac function. Females known to have anti-Ro or anti-La antibodies should be commenced on hydroxychloroquine to decrease risk of congenital heart block and neonatal lupus. ${ }^{41}$ For those previously on hydroxychloroquine, continuation throughout pregnancy is strongly advised due to association with improved renal outcomes and decreased risk of both flares and thrombotic events. ${ }^{42}$ Presence of anti-Ro antibodies will require serial fetal echocardiograms from Weeks 16-26 as per ACR guidelines. ${ }^{7}$ 
Pregnancy is a naturally prothrombotic event, requiring evaluation of need for additional anticoagulation during this period. All patients with SLE should be screened for $\mathrm{aPL}$ and if positive commenced on low-dose aspirin in the first trimester. Previous history of obstetric APS requires commencement of prophylactic heparin, while history of previous thrombotic APS requires therapeutic heparin during pregnancy. ${ }^{43}$ Positive aPL with no previous history can be managed with low-dose aspirin alone and close monitoring due to increased risk of pre-eclampsia.?

Active disease during pregnancy often requires a course of corticosteroids due to rapid onset of action. Frequency and duration of corticosteroid use should be limited due to potential increased risk of insulin resistance and infection. Evaluation of previous treatment and adherence must be considered prior to changing therapy. Hydroxychloroquine could be commenced but will take time to take effect. Azathioprine could also be considered. Current ACR guidelines recommend against the use of intravenous Ig (IVIG) due to limited evidence of benefit; however, it has been previously deemed compatible with all stages of pregnancy including breastfeeding. ${ }^{21}$ The European League Against Rheumatism (EULAR) guidelines also recommend consideration of IVIG in cases of severe refractory maternal disease in pregnancy. ${ }^{44}$ Belimumab is the first biologic agent approved for use in the treatment of SLE, but unfortunately pregnancy data is not yet available to comment on its safety.

\section{Rheumatoid Arthritis}

Research in RA has demonstrated that the inflammatory arthritis commonly goes into remission during the course of pregnancy. ${ }^{45}$ A recent meta-analysis reported improved disease activity in $60 \%$ of individuals during pregnancy, with $46.7 \%$ developing a flare in the postpartum period. ${ }^{46}$ This is driven by a series of modifications of the maternal immune system, including downregulation of effector $T$-cell activity with an increase in regulatory $T$ cells, to allow placentation and fetal growth. ${ }^{47}$ Following delivery, these immunomodulatory effects dissipate with the decrease of pregnancy hormones, resulting in postpartum flares.
Although many pregnant patients with RA will experience improvement of disease activity during pregnancy, disease activity must be monitored as part of routine care. Physiological changes of pregnancy resulting in increased erythrocyte sedimentation rate, $^{28}$ fatigue, weight gain, and anaemia can all affect commonly used indexes such as the Disease Activity Score in 28 joints (DAS28) and Health Assessment Questionnaire (HAQ). ${ }^{48}$ However, the DAS28-C-reactive protein 3, which excludes the global health score, has been shown to be the best clinical metric to detect active disease in patients with RA who are pregnant. ${ }^{49}$ Ongoing research has shown that additional indices also perform well in this population, including the Rheumatoid Arthritis Disease Activity Index (RADAI) and the Clinical Disease Activity Index (CDAI). ${ }^{50}$

Risk of flare during pregnancy is commonly associated with medication cessation and active disease prior to conception. ${ }^{23}$ There is no clear consensus on antibody presence and risk of flare in pregnancy. The Dutch Pregnancy-induced Amelioration of Rheumatoid Arthritis (PARA) study demonstrated a decreased risk of flare in people with RA who were pregnant and who did not carry autoantibodies. ${ }^{51}$ However, a smaller study showed females with RA with active disease in pregnancy had higher levels of anticitrullinated protein antibodies compared to those with lower disease activity. ${ }^{52}$ Further studies are needed to characterise the relationship between RA antibodies and risk of flare in pregnancy.

Flare in RA during pregnancy requires prompt management as early recognition can result in improved pain control and rapid inflammation reduction. Often rescue therapy in the form of corticosteroids is required for a limited period. Review of current therapy and compliance can be helpful to determine alternative treatment options. In a patient who had previously come off treatment or had not been on treatment, discussion of treatment initiation with a pregnancy-safe medication (Table 1 ) is required. ${ }^{7}$ 


\section{Spondyloarthropathy}

Spondyloarthropathy (SpA) includes both axial SpA and psoriatic arthritis. Unfortunately, research on pregnancy in SpA is not as abundant as other RMDs. Research in axial SpA has revealed $47.8 \%$ of patients experience active disease in pregnancy, ${ }^{53}$ with a peak in disease activity in the second trimester. Studies in psoriatic arthritis report quiescent disease during pregnancy with increased disease activity 6 months postpartum. ${ }^{54}$ Similar to other RMDs, stable disease at conception was associated with favourable pregnancy outcomes.

Pregnancy can mimic many symptoms of SpA, including low back pain, night pain, and fatigue, but currently there are no validated indices for monitoring SpA activity during pregnancy. In a disease where clinical assessment of the spine is a core component of monitoring disease, this is a significant limitation. This is further complicated by mechanical strain of the increasing mass of the gravid uterus on the spine during the course of the pregnancy and postpartum period. ${ }^{55}$ Further development of tools for monitoring SpA disease activity in pregnancy will provide much-needed insight into the effect of pregnancy on disease activity.

Ongoing development of national registers and collaboration between registers will allow for a rapid expansion of understanding of pregnancy in SpA. The European Network of Pregnancy Registers in Rheumatology (EuNeP) is a collaboration of four European national registers of patients with RMDs, formed with a focus on improving data collection on people with RMDs who are pregnant. ${ }^{56}$ This collaboration has identified challenges in collection and comparison of pregnancy data, in addition to recommendations for streamlining of pregnancy data going forward.

Non-steroidal anti-inflammatory drugs are used to treat flares in SpA; however, these should be avoided beyond 32 weeks due to risk of premature closure of the ductus arteriosus. ${ }^{57}$ Biologics such as TNF inhibitors are associated with stable disease in pregnancy and are recommended in pregnancy in the current ACR guidelines up to the third trimester, with the exception of certolizumab pegol, which can continue into the postpartum period. ${ }^{7}$

\section{Systemic Sclerosis}

Pregnancy in systemic sclerosis is uncommon as onset is typically after childbearing years. The primary concern in pregnancy is presence of PAH, which is the leading cause of maternal mortality.58 Those considering pregnancy should be evaluated for $\mathrm{PAH}$ as part of monitoring and counselled when considering pregnancy. In cases of established $\mathrm{PAH}$, patients should be strongly advised against pregnancy. ${ }^{59}$ Patients with systemic sclerosis who achieve pregnancy have a higher prevalence of adverse pregnancy outcomes (odds ratio [OR]: 1.9 for miscarriage; 3.8 for low birth weight; 2.4 for preterm birth). However, optimisation of disease control prior to conception decreases this risk, 60 although progression of disease during pregnancy was observed in $14.3 \%$. Close monitoring in specialist centres with support from obstetrics, rheumatology, and respiratory can aid in optimising outcomes.

Although now uncommon due to angiotensinconverting enzyme (ACE) inhibitors, there is a risk of scleroderma renal crisis in pregnancy. To avoid this, ACE inhibitors should be continued during pregnancy. ${ }^{61}$ Prior to conception, a trial period on an alternative antihypertensive agent could be considered to optimise blood pressure control. In cases of hypertension, despite alternative medication, ACE inhibitors should be restarted. Low-molecular-weight heparin can reduce thrombotic risk, while sildenafil and epoprostenol are additional therapeutic options in pregnancy. ${ }^{62}$ In cases of disease acceleration or rapid progression during pregnancy, additional life-saving therapies need to be considered, including preterm labour induction to allow use of additional medications not compatible with pregnancy.

\section{Vasculitis}

There are limited data on pregnancy in vasculitis because age of onset is typically beyond childbearing years, with a male predominance in most types of vasculitis. Disease activity in pregnancy varies by diagnosis, but all females with vasculitis should be considered for thromboprophylaxis during pregnancy. Pregnancy in these patients is associated with increased prevalence of pregnancy 
complications, especially pregnancy loss and preeclampsia;63 however, improved disease control prior to conception can improve outcomes. ${ }^{64}$

Patients with a new diagnosis or active disease prior to pregnancy are at higher risk of flares during pregnancy. Transitioning to pregnancysafe treatment is essential to ensure stable disease during pregnancy. This is especially important as flares in eosinophilic granulomatosis with polyangiitis and microscopic polyangiitis can have high fatality rates. ${ }^{65}$ A number of medications used to treat vasculitis are not compatible with pregnancy, leading to greater reliance on corticosteroids during this time. This should be limited when possible because of associations with adverse pregnancy outcomes, with increased reliance on low-risk medications including azathioprine and TNF inhibitors. ${ }^{64}$

\section{Inflammatory Myopathies}

The most common types of inflammatory myopathies are polymyositis and dermatomyositis. One study reported that $4-11 \%$ of females with these diseases had an onset age between 25 and 34 years. ${ }^{66}$ Pregnancy complications in this population were associated with active disease during pregnancy (OR: 435.4) and onset of disease prior to pregnancy (OR: 9.36). ${ }^{67}$ Relative risk of obstetric and fetal complications was 7.6 and 2.7, respectively, and were noted to occur more frequently following disease onset. ${ }^{68}$ Hypertensive disorders in pregnancy have also been found to occur more commonly (OR: 2.90). ${ }^{69}$ One analysis reported remission of disease during pregnancy, with active disease observed in $12.5 \%$, but with notably $56.2 \%$ reporting postpartum flares. ${ }^{70}$ Treatment in pregnancy is with IVIG and azathioprine, with limited use of glucocorticoids. ${ }^{11}$

\section{POSTPARTUM}

During the postpartum period, the mother's body again undergoes significant hormonal and physiological transitions as it recovers from the birth while adapting to breastfeeding.

This is compounded by the physical strain and sleep deprivation that comes with caring for a new infant. All of these factors can contribute to risk of postpartum flare. To minimise this risk, prompt re-evaluation of therapy is required.

A EULAR consensus statement in 2016 encouraged decisions on medication use during the postpartum period to maximise maternal benefit while minimising infant exposure. ${ }^{44}$ The 2020 ACR guidelines includes a section on postpartum use and lactation, ${ }^{7}$ highlighting the range of therapeutic options that can be safely offered to those breastfeeding to optimise disease control (Table 3).

\section{CONCLUSION}

Risk stratification and pregnancy planning are key to ensuring individuals with RMD have safe pregnancies with minimal risk of complications. This involves disease stabilisation, transition to pregnancy-compatible medications, assessment of autoantibody status, and evaluation of endorgan damage. Disease activity during the course of pregnancy varies by disease, with active disease in pregnancy associated with increased risk of pregnancy complications. The recent ACR guidelines detail the medications that are safe to use in pregnancy to control disease activity. Regular monitoring and communication between obstetrics and rheumatology is essential for maintaining stable disease in pregnancy and monitoring for complications.

\section{References}

1. Cooper GS, Stroehla BC. The epidemiology of autoimmune diseases. Autoimmun Rev. 2003;2(3):119-25.

2. Tincani $\mathrm{A}$ et al. Autoimmune diseases and pregnancy. Best Pract Res Clin Endocrinol Metab. 2019:33(6):101322.

3. Tincani A et al. Pregnancy in patients with autoimmune disease: a reality in 2016. Autoimmun Rev. 2016;15(10):975-7.

4. Bramham $\mathrm{K}$ et al. Pregnancy and renal outcomes in lupus nephritis: an update and guide to management. Lupus. 2012;21(12):127183.
5. Andreoli $L$ et al. EULAR recommendations for women's health and the management of family planning, assisted reproduction, pregnancy and menopause in patients with systemic lupus erythematosus and/ or antiphospholipid syndrome. Ann Rheum Dis. 2017;76(3):476-85. 
6. Østensen M. Preconception counseling. Rheum Dis Clin North Am. 2017;43(2):189-99.

7. Sammaritano LR et al. 2020 American college of rheumatology guideline for the management of reproductive health in rheumatic and musculoskeletal diseases. Arthritis Rheumatol. 2020;72(4):529-56.

8. Somers EC. Pregnancy and autoimmune diseases. Best Pract Res Clin Obstet Gynaecol. 2020;64:3-10.

9. Clowse ME et al. The impact of increased lupus activity on obstetric outcomes. Arthritis Rheum. 2005;52(2):514-21.

10. Soh MC, Nelson-Piercy C. High-risk pregnancy and the rheumatologist. Rheumatology (Oxford). 2015;54(4):572-87.

11. Andreoli $L$ et al. Disease activity assessment of rheumatic diseases during pregnancy: a comprehensive review of indices used in clinical studies. Autoimmun Rev. 2019;18(2):164-76

12. Sampson $M$ et al. An evidence-based practice guideline for the peer review of electronic search strategies. J Clin Epidemiol. 2009;62(9):944-52.

13. Hall JA et al. Pregnancy intention and pregnancy outcome: systematic review and meta-analysis. Matern Child Health J. 2017:21(3):670-4.

14. Bearak J et al. Global, regional, and subregional trends in unintended pregnancy and its outcomes from 1990 to 2014: estimates from a Bayesian hierarchical model. Lancet Glob Health. 2018;6(4):e380-9.

15. Goossens $\mathrm{J}$ et al. The prevalence of unplanned pregnancy ending in birth, associated factors, and health outcomes. Hum Reprod. 2016;31(12):2821-33.

16. Rajendran AEA et al. The importance of pregnancy planning in lupus pregnancies [abstract]. Arthritis \& Rheumatology. 2020;72.

17. Knight CL, Nelson-Piercy C. Management of systemic lupus erythematosus during pregnancy: challenges and solutions. Open Access Rheumatol. 2017;9:37-53.

18. Clowse MEB et al. Provider perceptions on the management of lupus during pregnancy: barriers to improved care. Lupus. 2019;28(1):8693.

19. Nahal SK et al. Safety issues and recommendations for successful pregnancy outcome in systemic lupus erythematosus. J Autoimmun 2018;93:16-23.

20. Sammaritano LR. Contraception and preconception counseling in women with autoimmune disease. Best Pract Res Clin Obstet Gynaecol. 2020;64:1123.

21. van den Brandt $\mathrm{S}$ et al. Risk factors for flare and treatment of disease flares during pregnancy in rheumatoid arthritis and axial spondyloarthritis patients. Arthritis Res Ther. 2017;19(1):64.

22. Flint $J$ et al. BSR and BHPR guideline on prescribing drugs in pregnancy and breastfeeding-part I: standard and biologic disease modifying anti-rheumatic drugs and corticosteroids. Rheumatology (Oxford). 2016;55(9):1693-7.

23. Desai RJ et al. Brief report: patterns and secular trends in use of immunomodulatory agents during pregnancy in women with rheumatic conditions. Arthritis Rheumatol. 2016;68(5):1183-9.

24. Hernández-Díaz S et al. Neural tube defects in relation to use of folic acid antagonists during pregnancy. Am J Epidemiol. 2001;153(10):961-8.

25. Izmirly PM et al. Maternal use of hydroxychloroquine is associated with a reduced risk of recurrent anti-SSA/Ro-antibody-associated cardiac manifestations of neonatal lupus. Circulation. 2012;126(1):76-82.

26. Tektonidou MG et al. EULAR recommendations for the management of antiphospholipid syndrome in adults. Ann Rheum Dis. 2019;78(10):1296-304

27. Schreiber K, Hunt BJ. Managing antiphospholipid syndrome in pregnancy. Thromb Res. 2019;181(Suppl 1):S41-6.

28. van den Broe NR, Letsky EA. Pregnancy and the erythrocyte sedimentation rate. Bjog. 2001;108(11):1164-7.

29. Cines DB, Levine LD. Thrombocytopenia in pregnancy. Blood. 2017;130(21):2271-7.

30. Pastore DEA et al. Systemic lupus erythematosus and pregnancy: the challenge of improving antenatal care and outcomes. Lupus. 2019;28(12):1417-26.

31. Tedeschi SK et al. Specific systemic lupus erythematosus disease manifestations in the six months prior to conception are associated with similar disease manifestations during pregnancy. Lupus. 2015;24(12):128392

32. Buyon JP et al. Assessing disease activity in SLE patients during pregnancy. Lupus. 1999;8(8):677-84.

33. Ruiz-Irastorza G et al. Measuring systemic lupus erythematosus activity during pregnancy: validation of the lupus activity index in pregnancy scale. Arthritis Rheum 2004;51(1):78-82.

34. Yee CS et al. The BILAG2004Pregnancy index is reliable for assessment of disease activity in pregnant SLE patients. Rheumatology (Oxford). 2012;51(10):1877-80.
35. Smyth A et al. A systematic review and meta-analysis of pregnancy outcomes in patients with systemic lupus erythematosus and lupus nephritis. Clin J Am Soc Nephrol. 2010;5(11):2060-8.

36. Gladman DD et al. The effect of lupus nephritis on pregnancy outcome and fetal and maternal complications. J Rheumatol. 2010;37(4):754-8.

37. Imbasciati E et al. Pregnancy in women with pre-existing lupus nephritis: predictors of fetal and maternal outcome. Nephrol Dial Transplant. 2009;24(2):519-25.

38. Lightstone L, Hladunewich MA. Lupus nephritis and pregnancy: concerns and management. Semin Nephrol. 2017;37(4):347-53

39. He $X$ et al. Clinical features of newonset systemic lupus erythematosus during pregnancy in Central China: a retrospective study of 68 pregnancies. Clin Rheumatol. 2021;40(6):2121-31.

40. Zhang $C$ et al. Clinical features of new-onset systemic lupus erythematosus in pregnant patients. $J$ Obstet Gynaecol Res. 2018;44(2):23440.

41. Izmirly PM et al. Evaluation of the risk of anti-SSA/Ro-SSB/La antibodyassociated cardiac manifestations of neonatal lupus in fetuses of mothers with systemic lupus erythematosus exposed to hydroxychloroquine. Ann Rheum Dis. 2010;69(10):1827-30.

42. Clowse ME et al. Hydroxychloroquine in lupus pregnancy. Arthritis Rheum. 2006:54(11):3640-7.

43. Mak A et al. Combination of heparin and aspirin is superior to aspirin alone in enhancing live births in patients with recurrent pregnancy loss and positive anti-phospholipid antibodies: a meta-analysis of randomized controlled trials and metaregression. Rheumatology (Oxford). 2010;49(2):281-8

44. Götestam Skorpen $C$ et al. The EULAR points to consider for use of antirheumatic drugs before pregnancy, and during pregnancy and lactation. Ann Rheum Dis. 2016;75(5):795-810.

45. Hench PS. The ameliorating effect of pregnancy on chronic atrophic (infection rheumatoid) arthritis, fibrositis and intermittent hydroarthrosis. Mayo Clinic Proc. 1938;13:161-7.

46. Jethwa $\mathrm{H}$ et al. Does rheumatoid arthritis really improve during pregnancy? a systematic review and metaanalysis. J Rheumatol. 2019;46(3):245-50.

47. Förger F, Villiger PM. Immunological adaptations in pregnancy that modulate rheumatoid arthritis disease activity. Nat Rev Rheumatol. 2020;16(2):113-22. 
48. de Man YA et al. Disease activity or remission of rheumatoid arthritis before, during and following pregnancy. Curr Opin Rheumatol. 2014;26(3):329-33.

49. de Man YA et al. Measuring disease activity and functionality during pregnancy in patients with rheumatoid arthritis. Arthritis Rheum. 2007:57(5):716-22.

50. Bermas BL et al. An evaluation of the patient-administered Rheumatoid Arthritis Disease Activity Index for assessing disease activity during pregnancy. Rheumatology (Oxford). 2017:56(12):2237-9.

51. de Man YA et al. Women with rheumatoid arthritis negative for anti-cyclic citrullinated peptide and rheumatoid factor are more likely to improve during pregnancy, whereas in autoantibody-positive women autoantibody levels are not influenced by pregnancy. Ann Rheum Dis. 2010;69(2):420-3

52. Förger $\mathrm{F}$ et al. Pregnancy mediated improvement of rheumatoid arthritis. Swiss Med Wkly. 2012;142:w13644.

53. Maguire S et al. Pregnancy in axial spondyloarthropathy: A systematic review \& meta-analysis. Semin Arthritis Rheum. 2020;50(6):1269-79.

54. Ursin $\mathrm{K}$ et al. Psoriatic arthritis disease activity during and after pregnancy: a prospective multicenter Study. Arthritis Care Res (Hoboken) 2019;71(8):1092-100.

55. Østensen $\mathrm{M}$ et al. A prospective study of pregnant patients with rheumatoid arthritis and ankylosing spondylitis using validated clinical instruments. Ann Rheum Dis. 2004;63(10):1212-7.

56. Meissner $Y$ et al. European network of pregnancy registers in rheumatology (EuNeP)-an overview of procedures and data collection. Arthritis Res Ther. 2019;21(1):241.

57. Østensen $\mathrm{M}$ et al. Anti-inflammatory and immunosuppressive drugs and reproduction. Arthritis Res Ther. 2006;8(3):209.

58. Clark KE et al. Systemic sclerosis in pregnancy. Obstet Med. 2020;13(3):105-11.

59. Martin SR, Edwards A. Pulmonary hypertension and pregnancy. Obstet Gynecol. 2019;134(5):974-87.

60. Blagojevic J et al. Pregnancy in systemic sclerosis: results of a systematic review and Metaanalysis. J Rheumatol. 2020;47(6):881-7.

61. Baethge BA, Wolf RE. Successfu pregnancy with scleroderma renal disease and pulmonary hypertension in a patient using angiotensin converting enzyme inhibitors. Ann Rheum Dis. 1989;48(9):776-8.

62. Lidar M, Langevitz P. Pregnancy issues in scleroderma. Autoimmun Rev. 2012;11(6-7):A515-9.

63. Nguyen $\vee$ et al. Pregnancy outcomes in women with primary systemic vasculitis: a retrospective study. J Matern Fetal Neonatal Med. 2019:1-7.
64. Machen L, Clowse ME Vasculitis and pregnancy. Rheum Dis Clin North Am. 2017;43(2):239-47.

65. Sangle SR et al. Pregnancy outcome in patients with systemic vasculitis: a single-centre matched case-control study. Rheumatology (Oxford). 2015;54(9):1582-6.

66. Ohta A et al. Age at onset and gender distribution of systemic lupus erythematosus, polymyositis/ dermatomyositis, and systemic sclerosis in Japan. Mod Rheumatol. 2013;23(4):759-64.

67. Zhong $Z$ et al. Pregnancy in polymyositis or dermatomyositis: retrospective results from a tertiary centre in China. Rheumatology (Oxford). 2017;56(8):1272-5

68. Gupta L et al. Outcomes of pregnancy in women with inflammatory myositis: a cohort study from India. J Clin Rheumatol. 2020;26(5):165-8.

69. Kolstad KD et al. Pregnancy outcomes in adult patients with dermatomyositis and polymyositis. Semin Arthritis Rheum. 2018;47(6):865-9.

70. Pinal-Fernandez I et al. "Pregnancy in adult-onset idiopathic inflammatory myopathy": report from a cohort of myositis patients from a single center. Semin Arthritis Rheum. 2014;44(2):234-40.

71. Munira S, Christopher-Stine L. Pregnancy in myositis and scleroderma. Best Pract Res Clin Obstet Gynaecol. 2020;64:59-67. 